\title{
Is the Sedimentation Sign Associated With Spinal Stenosis Surgical Treatment Effect in SPORT?
}

\author{
Rachel A. Moses, MD, ${ }^{*}$ Wenyan Zhao, PhD, ${ }^{*}+$ Lukas P. Staub, PhD, $\neq$ \\ Markus Melloh, MD, DMedSc, MPH, PhD, MBA, § Thomas Barz, MD, $\uparrow$ and Jon D. Lurie, MD, MS*+\|
}

\begin{abstract}
Study Design. Subgroup analysis of the lumbar spinal stenosis (LSS) without degenerative spondylolisthesis diagnostic cohort of the Spine Patient Outcomes Research Trial multicenter randomized clinical trial with a concurrent observational cohort.
\end{abstract}

Objective. To determine if sedimentation sign on magnetic resonance image can help with LSS treatment decisions.

Summary of Background Data. LSS is one of the most common reasons for surgery in the US elderly, but there is a dearth of reliable diagnostic tools that give a clear indication for surgery. Recent studies have suggested that positive sedimentation sign on magnetic resonance image may be a possible prognostic indicator.

Methods. All patients with LSS in both the randomized and observational cohorts had imaging-confirmed stenosis, were surgical candidates, and had neurogenic claudication for at least 12 weeks prior to enrollment. Patients were categorized as "mild," "moderate," or "severe" according to stenosis severity. Of the 654 patients with LSS enrolled in Spine Patient Outcomes Research Trial, complete T2-weighted axial and sagittal digitized images of 115 patients were available for retrospective review. An independent orthopedic spine surgeon evaluated these deidentified Digital Imaging and Communications in Medicine files for the sedimentation sign.

From the *Department of Orthopaedics, Dartmouth-Hitchcock Medical Center, Lebanon, $\mathrm{NH}$; +The Dartmouth Institute for Health Policy and Clinical Practice, Geisel School of Medicine, Lebanon, $\mathrm{NH}$; \#Institute for Evaluative Research in Orthopaedic Surgery, University of Bern, Bern, Switzerland; $\S$ Centre for Health Sciences, Zurich University of Applied Sciences, Winterthur, Switzerland; I Department of Orthopaedic Surgery, Asklepios Klinikum Uckermark, Schwedt, Germany; and |Department of Medicine, Geisel School of Medicine, Lebanon, NH.

Acknowledgment date: November 13, 2013. First revision date: April 23, 2014. Second revision date: June 27, 2014. Acceptance date: June 29, 2014.

The manuscript submitted does not contain information about medical device(s)/drug(s).

The National Institute of Arthritis and Musculoskeletal and Skin Diseases (U01-AR45444) and the Office of Research on Women's Health, the National Institutes of Health, and the National Institute of Occupational Safety and Health, the Centers for Disease Control and Prevention funds were received in support of this work. Dr Moses is partially funded by NIAMS ARRA supplement to the Ruth L. Kirschstein NRSA (3T32AR049710-07S1). The Multidisciplinary Clinical Research Center in Musculoskeletal Diseases is funded by NIAMS (P60-AR048094 and P60-AR062799).

Relevant financial activities outside the submitted work: consultancy, stocks. Address correspondence and reprint requests to Jon D. Lurie, MD, MS, Departments of Medicine, Orthopaedics and the Dartmouth Institute, Dartmouth-Hitchcock Medical Center, Lebanon, NH 03756; E-mail: jonathan.d.lurie@hitchcock.org or tamara.s.morgan@dartmouth.edu

DOI: 10.1097/BRS.0000000000000672

Spine
Results. Sixty-six percent (76/115) of patients were found to have a positive sedimentation sign. Those with a positive sedimentation sign were more likely to have stenosis at L2-L3 (33\% vs. $10 \% P=$ $0.016)$ or L3-L4 76\% vs. 51\%, $P=0.012)$, and to have severe $(72 \%$ vs. $33 \%, P<0.0001)$ central stenosis $(93 \%$ vs. $67 \% P<0.001)$ at 2 or more concurrent levels ( $57 \%$ vs. $18 \%, P=0.01)$. In multivariate models, the surgical treatment effect was significantly larger in the positive sedimentation sign group for Oswestry Disability Index $(-16$ vs. $-7 ; P=0.02)$.

Conclusion. A positive sedimentation sign was associated with a small but significantly greater surgical treatment effect for Oswestry Disability Index in patients with symptomatic LSS, after adjusting for other demographic and imaging features. These findings suggest that positive sedimentation sign may potentially be a useful adjunct to help guide an informed treatment choice regarding surgery for LSS.

Key words: lumbar spinal stenosis, sedimentation sign, MRI, shared decision making, surgical treatment.

Level of Evidence: 2

Spine 2015;40:129-136

irst described by Verbiest in $1947,{ }^{1}$ lumbar spinal ste- nosis (LSS) has become one of the most common surgically treated ailments affecting our aging population. ${ }^{2,3}$ Despite this fact, there have yet to be clear treatment indications for this disease, partly due to a lack of reliable diagnostic tools. ${ }^{4-10}$ In addition to clinical diagnostic tests, ${ }^{11-15}$ cross-sectional spinal canal narrowing has become a commonly accepted imaging criteria of LSS, ${ }^{16,17}$ yet, several studies have shown that there can be little correlation between anatomical narrowing, clinical symptoms, and treatment outcomes. ${ }^{5,11,7,8,15,17-22}$

Recently, Barz et $a l^{23}$ introduced the sedimentation sign on magnetic resonance image (MRI) as a possible aid in decision making for LSS. Normally, in the supine position the lumbar nerve roots should settle to the posterior spinal canal due to gravity and the absence of constrictive force. A positive sedimentation sign, however, is evident when a patient lying supine has the absence of posterior nerve root settling. 1,14,23,24 A previous study by Barz et $a^{23}$ demonstrated the ability of the sedimentation sign to discriminate between known cases of symptomatic central LSS and controls with nonspecific low back pain. An observational follow-up study by the same 
group showed that, in patients treated with decompression surgery, the sign does not seem to predict surgical outcome, whereas in nonoperatively treated patients, a positive sign is associated with more limited improvement. ${ }^{25}$ This evidence indicates that the sedimentation sign may provide information about prognosis in patients who do not proceed to surgery and/or help to identify patients more likely to benefit from surgery. However, to date no studies have provided unbiased estimates of the relationship between a positive sedimentation sign and treatment outcomes in patients with LSS.

The Spine Patient Outcomes Research Trial (SPORT), a multicenter randomized clinical trial with a concurrent observational cohort, demonstrated better outcomes for patients with LSS treated surgically than those treated nonoperatively; however, 2 years after surgery only $63 \%$ report major symptomatic improvement, whereas almost 30\% of patients treated nonoperatively report a similar level of improvement. ${ }^{26}$ Improved ability to identify patients more or less likely to benefit from surgical intervention would be an important aid to clinical decision making. To this end, we performed a subgroup analysis on the SPORT LSS cohort; we used baseline MR images from patients with LSS enrolled in SPORT to evaluate the association between a positive sedimentation sign and surgical treatment effect, defined as the difference between the average change in the surgical group minus the average change in the nonoperative group. In consideration of the observational evidence available, we hypothesized that a positive sedimentation sign may predict poorer outcomes with nonoperative treatment and/or relatively improved outcomes with surgery, thus demonstrating a greater surgical treatment effect. Our analysis focuses on the difference in outcome between the surgical group compared with the nonoperative group, which is the surgical treatment effect, because it is the key parameter in understanding the relative benefit of surgery, which in turn is the crucial piece of information in decision making for a patient weighing the choice between those 2 options.

\section{MATERIALS AND METHODS}

SPORT was a multicenter investigation that obtained prospective data from 13 spine clinics contained in 11 states. All included patients had to be surgical candidates, have a history of neurogenic claudication for a period of at least 12 weeks, and have cross-sectional imaging demonstrating stenosis. Stenosis severity was categorized as mild: dural area compromise of $1 / 3$ or more expected; moderate: dural area compromise between 1/3 and 2/3 expected; and severe: a dural area compromise of more than $2 / 3$ expected..$^{27}$ Patients with lumbar instability and degenerative spondylolisthesis were excluded from this cohort. Standard posterior decompression laminectomy comprised surgical management. ${ }^{28}$ Nonoperative interventions included prescribed physical activity, counseling, steroid epidural injections, opioids, and nonsteroidal antiinflammatory drugs. ${ }^{28,29}$

The study's primary outcome measures included the 36-Item Short Form General Health Survey (SF-36) Physical Function and Bodily Pain scales, ${ }^{30,31}$ the stenosis bothersomeness index (SBI), ${ }^{32,33}$ and the modified Oswestry Disability Index (ODI). ${ }^{34}$ The SF-36 is scored on a scale of 0 to 100 with larger scores indicating better function and less pain. The SBI scores range from 0 to 24 with lower scores indicating less severe symptoms, and the ODI is scored on a scale of 0 to 100 and similarly, lower scores indicate a better outcome.

A total of 654 patients with LSS were enrolled in SPORT. ${ }^{26,27}$ Of these patients, complete T2-weighted axial and sagittal digitized images of 115 patients were available for retrospective review, which were deidentified and electronically uploaded as Digital Imaging and Communications in Medicine files. ${ }^{27}$ These images were provided to an independent orthopedic spine surgeon on individual compact discs and eFilm Light software was used to view these images (Merge Technologies, Milwaukee, WI). Each patient's T2-weighted axial images were evaluated for the presence of a positive sedimentation sign. Following the original description by Barz et al, ${ }^{23}$ "a positive sedimentation sign is defined
Negative sedimentation sign: Normal nerve root sedimentation
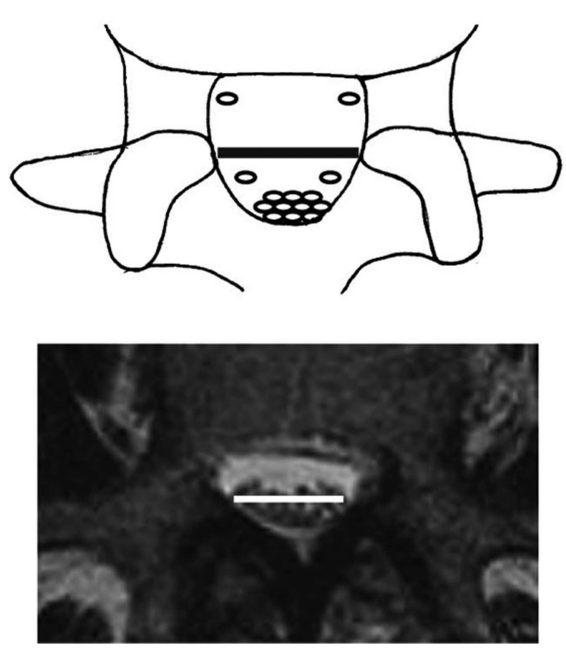

Positive sedimentation sign:

Absent nerve root sedimentation
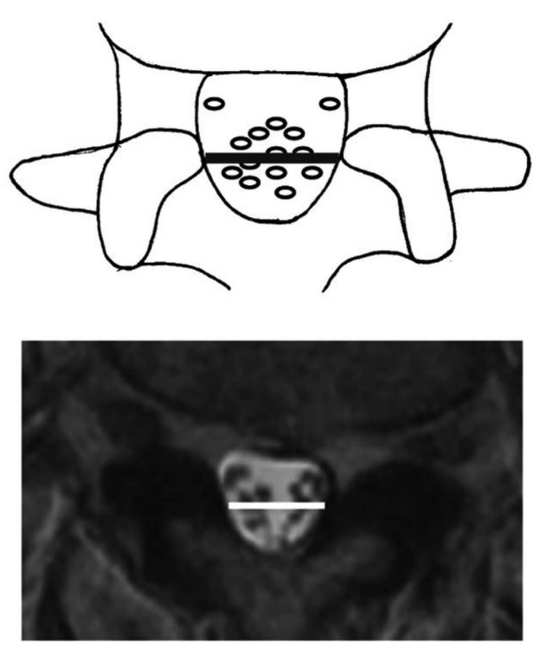

Figure 1. Left: negative sedimentation sign example (normal) and right: positive sedimentation sign (abnormal). Reproduced with permission from the study by Barz et al. ${ }^{23}$ 


\begin{tabular}{|c|c|c|c|}
\hline \multicolumn{4}{|c|}{$\begin{array}{l}\text { TABLE 1. Baseline Demographic Characteristics, } \\
\text { Comorbidities, and Health Status } \\
\text { Measures of the Patients According to } \\
\text { Whether the Patient Had Nerve Root } \\
\text { Sedimentation Sign }\end{array}$} \\
\hline \multirow[b]{2}{*}{ Characteristic } & \multicolumn{2}{|c|}{ Sedimentation Sign } & \multirow[b]{2}{*}{$\boldsymbol{P}$} \\
\hline & $\begin{array}{l}\text { Negative } \\
(\mathrm{n}=39)^{*}\end{array}$ & $\begin{array}{c}\text { Positive } \\
(\mathbf{n}=76)^{*}\end{array}$ & \\
\hline Age, mean (SD), yr & $61.4(11.1)$ & $65.7(12.9)$ & 0.075 \\
\hline Females, no. $(\%)$ & $19(49)$ & $32(42)$ & 0.63 \\
\hline $\begin{array}{l}\text { Ethnicity, non-Hispanic, no. } \\
(\%) \dagger\end{array}$ & $34(87)$ & $70(92)$ & 0.61 \\
\hline Race, Caucasian, no. $(\%) \dagger$ & $29(74)$ & $63(83)$ & 0.40 \\
\hline $\begin{array}{l}\text { Education, at least some } \\
\text { college, no. }(\%)\end{array}$ & $28(72)$ & $42(55)$ & 0.13 \\
\hline Income, under $\$ 50,000$ & $8(21)$ & $16(21)$ & 0.86 \\
\hline Married, no. (\%) & $27(69)$ & $50(66)$ & 0.87 \\
\hline Work status, no. (\%) & & & 0.41 \\
\hline Full or part time & $18(46)$ & $24(32)$ & \\
\hline Disabled & $2(5)$ & $7(9)$ & \\
\hline Retired & $16(41)$ & $35(46)$ & \\
\hline Other & $3(8)$ & $10(13)$ & \\
\hline $\begin{array}{l}\text { Disability compensation, } \\
\text { no. }(\%) \ddagger\end{array}$ & $3(8)$ & $3(4)$ & 0.68 \\
\hline BMI, mean $(\mathrm{SD}) \S$ & $28.3(4.3)$ & $29.1(5.5)$ & 0.42 \\
\hline Smoker, no. (\%) & $8(21)$ & $10(13)$ & 0.45 \\
\hline \multicolumn{4}{|l|}{ Comorbidities, no. $(\%)$} \\
\hline Hypertension & $14(36)$ & $40(53)$ & 0.13 \\
\hline Diabetes & $5(13)$ & $14(18)$ & 0.62 \\
\hline Osteoporosis & $8(21)$ & $\mathrm{t} 4(5)$ & 0.027 \\
\hline Heart problem & $7(18)$ & $19(25)$ & 0.54 \\
\hline Stomach problem & $10(26)$ & $13(17)$ & 0.40 \\
\hline $\begin{array}{l}\text { Bowel or intestinal } \\
\text { problem }\end{array}$ & $5(13)$ & $4(5)$ & 0.29 \\
\hline Depression & $5(13)$ & $10(13)$ & 0.81 \\
\hline Joint problem & $20(51)$ & $37(49)$ & 0.95 \\
\hline Other $₫$ & $13(33)$ & $27(36)$ & 0.98 \\
\hline $\begin{array}{l}\text { Time since most recent } \\
\text { episode }>6 \text { mo, no. }(\%)\end{array}$ & $23(59)$ & $36(47)$ & 0.33 \\
\hline \multicolumn{4}{|l|}{ SF-36 scores, mean (SD)\| } \\
\hline $\mathrm{BP}$ & 32.7 (18.6) & $32.4(18)$ & 0.95 \\
\hline $\mathrm{PF}$ & $44.7(26.4)$ & 36.7 (22.6) & 0.091 \\
\hline PCS & $51.4(10.6)$ & $49.5(12.9)$ & 0.44 \\
\hline MCS & $32.1(8.5)$ & $29.6(9.3)$ & 0.17 \\
\hline $\mathrm{ODI}^{* *}$ & $42.9(22.4)$ & $38.9(18.7)$ & 0.32 \\
\hline $\begin{array}{l}\text { Stenosis frequency index } \\
(0-24)+\dagger\end{array}$ & $14.2(6.6)$ & $12.7(6.3)$ & 0.24 \\
\hline
\end{tabular}

(Continued)

\section{TABLE 1. (Continued)}

\begin{tabular}{|c|c|c|c|}
\hline \multirow[b]{2}{*}{ Characteristic } & \multicolumn{2}{|c|}{ Sedimentation Sign } & \multirow[b]{2}{*}{$\boldsymbol{P}$} \\
\hline & $\begin{array}{l}\text { Negative } \\
(\mathbf{n}=39)^{*}\end{array}$ & $\begin{array}{l}\text { Positive } \\
(\mathbf{n}=76)^{*}\end{array}$ & \\
\hline $\begin{array}{l}\text { Stenosis bothersomeness } \\
\text { index }\end{array}$ & $13.7(6.5)$ & $13(5.9)$ & 0.57 \\
\hline $\begin{array}{l}\text { Back pain bothersomeness } \\
\text { index } \S \S\end{array}$ & $3.9(1.9)$ & $4.1(1.7)$ & 0.47 \\
\hline $\begin{array}{l}\text { Leg pain bothersomeness } \\
\text { index } \boldsymbol{\uparrow}\end{array}$ & $4.1(1.8)$ & $4.1(1.6)$ & 0.88 \\
\hline $\begin{array}{l}\text { Patient very dissatisfied with } \\
\text { symptoms, no. (\%) }\end{array}$ & $25(64)$ & $48(63)$ & 0.92 \\
\hline $\begin{array}{l}\text { Patient's self-assessed health } \\
\text { trend, no. }(\%)\end{array}$ & & & 0.40 \\
\hline Problem getting better & $3(8)$ & $10(13)$ & \\
\hline $\begin{array}{l}\text { Problem staying about the } \\
\text { same }\end{array}$ & $10(26)$ & $24(32)$ & \\
\hline Problem getting worse & $26(67)$ & $40(53)$ & \\
\hline $\begin{array}{l}\text { Treatment preference, } \\
\text { no. }(\%)\end{array}$ & & & 0.55 \\
\hline $\begin{array}{l}\text { Preference for nonsurgical } \\
\text { treatment }\end{array}$ & $14(36)$ & $35(46)$ & \\
\hline Not sure & $12(31)$ & $18(24)$ & \\
\hline Preference for surgery & $13(33)$ & $23(30)$ & \\
\hline $\begin{array}{l}\text { Neurogenic claudication, } \\
\text { no. }(\%)\end{array}$ & $34(87)$ & $65(86)$ & 0.97 \\
\hline $\begin{array}{l}\text { Pain on straight leg raising } \\
\text { or femoral nerve tension } \\
\text { sign, no. }(\%)\end{array}$ & $7(18)$ & $7(9)$ & 0.29 \\
\hline $\begin{array}{l}\text { Dermatomal pain radiation, } \\
\text { no. }(\%)\end{array}$ & $35(90)$ & $60(79)$ & 0.24 \\
\hline $\begin{array}{l}\text { Any neurological deficit, } \\
\text { no. }(\%)\end{array}$ & $25(64)$ & $40(53)$ & 0.33 \\
\hline $\begin{array}{l}\text { Asymmetric depressed } \\
\text { reflexes }\end{array}$ & $9(23)$ & $18(24)$ & 0.87 \\
\hline $\begin{array}{l}\text { Asymmetric decrease in } \\
\text { sensory reflexes }\end{array}$ & $15(38)$ & $17(22)$ & 0.11 \\
\hline $\begin{array}{l}\text { Asymmetric motor } \\
\text { weakness }\end{array}$ & $13(33)$ & $23(30)$ & 0.90 \\
\hline
\end{tabular}

Stenosis levels, no. (\%)

\begin{tabular}{|l|c|c|c|}
\hline L2-L3 & $4(10)$ & $25(33)$ & 0.016 \\
\hline L3-L4 & $20(51)$ & $58(76)$ & 0.012 \\
\hline L4-L5 & $35(90)$ & $71(93)$ & 0.74 \\
\hline L5-S1 & $10(26)$ & $18(24)$ & 1 \\
\hline $\begin{array}{c}\text { Stenotic levels (moderate/ } \\
\text { severe) }\end{array}$ & & & 0.01 \\
\hline None & $1(3)$ & $0(0)$ & \\
\hline 1 & $20(51)$ & $19(25)$ & \\
\hline 2 & $14(36)$ & $37(49)$ & \\
\hline $3+$ & $4(10)$ & $20(26)$ & \\
\hline
\end{tabular}




\begin{tabular}{|c|c|c|c|}
\hline \multirow[b]{2}{*}{ Characteristic } & \multicolumn{2}{|c|}{ Sedimentation Sign } & \multirow[b]{2}{*}{$\boldsymbol{P}$} \\
\hline & $\begin{array}{l}\text { Negative } \\
(\mathrm{n}=39)^{*}\end{array}$ & $\begin{array}{c}\text { Positive } \\
(\mathbf{n}=76)^{*} \\
\end{array}$ & \\
\hline \multicolumn{4}{|l|}{ Stenosis locations, no. $(\%)$} \\
\hline Central & $26(67)$ & $71(93)$ & $<0.001$ \\
\hline Lateral recess & $32(82)$ & $65(86)$ & 0.83 \\
\hline Neuroforamen & $12(31)$ & $19(25)$ & 0.66 \\
\hline Stenosis severity, no. (\%) & & & $<0.001$ \\
\hline Mild & $1(3)$ & $0(0)$ & \\
\hline Moderate & $25(64)$ & $21(28)$ & \\
\hline Severe & $13(33)$ & $55(72)$ & \\
\hline Spinal instability|||| & $0(0)$ & $0(0)$ & $\ldots$ \\
\hline Underwent surgery*** & $28(72)$ & $47(62)$ & 0.39 \\
\hline \multicolumn{4}{|c|}{$\begin{array}{l}\text { *Total } 115 \text { patients, who had MRI/information of nerve root sedimentation } \\
\text { sign and had at least } 1 \text { follow-up through } 4 \text { yr, are in the current analysis. }\end{array}$} \\
\hline \multicolumn{4}{|c|}{$\begin{array}{l}\text { \#This category includes patients who were receiving or had applications } \\
\text { pending for workers compensation, social security, or other compensation. }\end{array}$} \\
\hline \multicolumn{4}{|c|}{$\begin{array}{l}\text { \$The body mass index is the weight in kilograms divided by the square of } \\
\text { the height in meters. }\end{array}$} \\
\hline \multicolumn{4}{|c|}{$\begin{array}{l}\text { TOther denotes problems related to stroke, cancer, lung, fibromyalgia, CFS, } \\
\text { PTSD, alcohol, drug dependency, liver, kidney, blood vessel, nervous system, } \\
\text { migraine, and anxiety. }\end{array}$} \\
\hline \multicolumn{4}{|c|}{$\begin{array}{l}\| \text { The SF- } 36 \text { scores range from } 0 \text { to 100, with higher score indicating less } \\
\text { severe symptoms. }\end{array}$} \\
\hline \multicolumn{4}{|c|}{$\begin{array}{l}\text { **The } O D I \text { ranges from } 0 \text { to } 100 \text {, with lower scores indicating less severe } \\
\text { symptoms. }\end{array}$} \\
\hline \multicolumn{4}{|c|}{$\begin{array}{l}++ \text { The stenosis frequency index ranges from } 0 \text { to } 24 \text {, with lower scores } \\
\text { indicating less severe symptoms. }\end{array}$} \\
\hline \multicolumn{4}{|c|}{$\begin{array}{l}\neq \neq \text { The stenosis bothersomeness index ranges from } 0 \text { to } 24 \text {, with lower scores } \\
\text { indicating less severe symptoms. }\end{array}$} \\
\hline \multicolumn{4}{|c|}{$\begin{array}{l}\S \S \text { The low back pain bothersomeness index ranges from } 0 \text { to } 6 \text {, with lower } \\
\text { scores indicating less severe symptoms. }\end{array}$} \\
\hline \multicolumn{4}{|c|}{$\begin{array}{l}\text { 99The leg pain bothersomeness index ranges from } 0 \text { to } 6 \text {, with lower scores } \\
\text { indicating less severe symptoms. }\end{array}$} \\
\hline \multicolumn{4}{|c|}{$\begin{array}{l}\|\| \text { Spinal instability is defined as a change of more than } 10^{\circ} \text { of angulation } \\
\text { or more than } 4 \mathrm{~mm} \text { of translation of the vertebrae between flexion and } \\
\text { extension of the spine. }\end{array}$} \\
\hline \multicolumn{4}{|c|}{$\begin{array}{l}\text { ****Patients who underwent surgery were classified according to whether } \\
\text { they underwent surgical treatment during the first } 4 \text { yr of enrollment. }\end{array}$} \\
\hline \multicolumn{4}{|c|}{$\begin{array}{l}\text { BMI indicates body mass index; BP, bodily pain; PF, physical functioning; } \\
\text { PCS, physical component summary; MCS, mental component summary; } \\
\text { ODI, Oswestry Disability Index; SF-36, 36-Item Short Form General Health } \\
\text { Survey; MRI, magnetic resonance imaging; CFS, chronic fatigue syndrome; } \\
\text { PTSD, post-traumatic stress disorder. }\end{array}$} \\
\hline
\end{tabular}

as the absence of nerve root sedimentation in at least 1 transverse MR image, at a level above or below, disregarding the location of the scan within the level and its proximity to the maximal stenosis" (Figure 1). As a result, the sign is defined as overall positive or negative for a given patient rather than level by level within a patient.

\section{Statistical Analysis}

Baseline characteristics of patients with and without a positive sedimentation sign were compared using a $\chi^{2}$ test for categorical variables and $t$ tests for continuous variables. The primary analysis compared surgical and nonoperative treatments using changes from baseline at each follow-up, with a mixed effects longitudinal regression model including a random individual effect to account for correlation between repeated measurements within individuals. Because of high rates of treatment crossover, analyses were based on treatments actually received in the combined randomized and observational cohorts as described in the previous text. ${ }^{26,28}$

To adjust for potential confounding, baseline variables that were significantly different between the 2 subgroups (those with and without a positive sedimentation sign) were included as adjusting covariates in the models. In addition, age, sex, and baseline outcome scores (for SF-36, ODI, and SBI) were included in all longitudinal outcome models. Across the 4-year follow-up, overall comparisons of the area under the curve between these subgroups were made using a Wald test. Computations were done using SAS PROC MIXED procedure (SAS version 9.2 Windows XP Pro; SAS Institute Inc., Cary, NC). Statistical significance was defined as $P<0.05$ based on a 2-sided hypothesis test with no adjustments made for multiple comparisons.

\section{RESULTS}

The 115 baseline MRIs were reviewed by an independent reader who was blinded to treatment group and outcome. Sixty-six percent $(76 / 115)$ of patients were found to have a positive sedimentation sign. Table 1 depicts the comparison of baseline demographics between the 2 subgroups demonstrating that individuals with a positive sedimentation sign were more likely to have stenosis at either L2-L3 (33\% vs. 10\%, $P=0.016)$ or L3-L4 (76\% vs. $51 \%, P=0.012)$, central stenosis $(93 \%$ vs. $67 \%, P<0.001)$, severe stenosis $(72 \%$ vs. $33 \%, P<0.0001)$, and have 2 or more concurrent stenotic levels $(57 \%$ vs. $18 \%, P=0.01)$.

Table 2 compares the surgical procedures and complications between the 2 groups. A total of 75 patients in this subgroup analysis underwent surgery, 47 of 76 (62\%) of those with a positive sedimentation sign and 28 of $39(72 \%)$ of those with a negative sign. There were no statistically significant differences in operative treatment or complication rates between the 2 subgroups.

The 4-year time-weighted average of outcome measures in the 2 subgroups is summarized in Table 3 . In general, the surgical treatment effect was larger in the positive sedimentation sign group for all outcomes. This was statistically significant for ODI ( -16 vs. $-7 ; P=0.02)$; SBI $(-5.7$ vs. $-3.2 ; P=$ $0.09)$, SF-36 bodily pain (15.4 vs. $11.5 ; P=0.44)$, and SF-36 physical function (17.7 vs. $15.1 ; P=0.61)$ were not significantly different. These analyses were controlled for age, sex, baseline outcome score, osteoporosis, stenosis level, stenosis location (central $v s$. foraminal), and stenosis severity.

\section{DISCUSSION}

Determining the optimal treatment intervention for patients with symptomatic LSS can be difficult because the degree and location of anatomical narrowing may not correlate with 


\begin{tabular}{|c|c|c|c|}
\hline \multirow[b]{2}{*}{ Characteristic } & \multicolumn{2}{|c|}{ Sedimentation Sign } & \multirow[b]{2}{*}{$\boldsymbol{P}$} \\
\hline & $\begin{array}{l}\text { Negative } \\
(\mathbf{n}=28)^{*}\end{array}$ & $\begin{array}{l}\text { Positive } \\
(\mathrm{n}=47)^{*}\end{array}$ & \\
\hline Procedure & & & 0.67 \\
\hline Decompression only & $24(89)$ & $41(93)$ & \\
\hline Noninstrumented fusion & $0(0)$ & $0(0)$ & \\
\hline Instrumented fusion & $3(11)$ & $3(7)$ & \\
\hline Multilevel fusion & $1(4)$ & $3(6)$ & 1 \\
\hline \multicolumn{4}{|l|}{ Decompression level, no. (\%) } \\
\hline L2-L3 & $5(19)$ & $24(51)$ & 0.012 \\
\hline L3-L4 & $17(65)$ & $39(83)$ & 0.15 \\
\hline L4-L5 & $24(92)$ & $45(96)$ & 0.61 \\
\hline L5-S1 & $14(54)$ & $16(34)$ & 0.14 \\
\hline Levels decompressed, no. (\%) & & & 0.026 \\
\hline None & $2(7)$ & $0(0)$ & \\
\hline 1 & $4(14)$ & $8(17)$ & \\
\hline 2 & $13(46)$ & $11(23)$ & \\
\hline $3+$ & $9(32)$ & $28(60)$ & \\
\hline $\begin{array}{l}\text { Operation time, min, mean } \\
\text { (SD) }\end{array}$ & $\begin{array}{l}137.4 \\
(88.1) \\
\end{array}$ & $\begin{array}{l}151.5 \\
(73.5) \\
\end{array}$ & 0.46 \\
\hline Blood loss, mL, mean (SD) & $\begin{array}{c}480.3 \\
(1001.3)\end{array}$ & $\begin{array}{c}386.6 \\
(419.8)\end{array}$ & 0.58 \\
\hline
\end{tabular}

Blood replacement, no. (\%)

\begin{tabular}{|c|c|c|c|}
\hline Intraoperative replacement & $3(11)$ & $6(13)$ & 1 \\
\hline Postoperative transfusion & $2(7)$ & $3(7)$ & 1 \\
\hline $\begin{array}{c}\text { Length of hospital stay, d, } \\
\text { mean (SD) }\end{array}$ & $3.4(2.3)$ & $3.2(1.7)$ & 0.65 \\
\hline
\end{tabular}

Intraoperative complications, no. (\%)†

\begin{tabular}{|l|c|c|c|}
\hline Dural tear/spinal fluid leak & $2(7)$ & $5(11)$ & 0.71 \\
\hline None & $26(93)$ & $42(89)$ & 0.71 \\
\hline
\end{tabular}

Postoperative complications/events, no. (\%)‡

\begin{tabular}{|c|c|c|c|}
\hline Wound hematoma & $1(4)$ & $0(0)$ & 0.38 \\
\hline Other & $1(4)$ & $3(7)$ & 1 \\
\hline None & $25(89)$ & $40(87)$ & 1 \\
\hline $\begin{array}{l}\text { Postoperative mortality (death } \\
\text { within } 6 \text { wk of surgery), } \\
\text { no. }(\%)\end{array}$ & $0(0)$ & $0(0)$ & \\
\hline $\begin{array}{l}\text { Postoperative mortality (death } \\
\text { within } 3 \text { mo of surgery), } \\
\text { no. }(\%)\end{array}$ & $0(0)$ & $0(0)$ & \\
\hline $\begin{array}{l}\text { Additional surgical proce- } \\
\text { dures (1-yr rate), no. (\%)§ }\end{array}$ & $1(4)$ & $0(0)$ & 0.195 \\
\hline
\end{tabular}

(Continued)

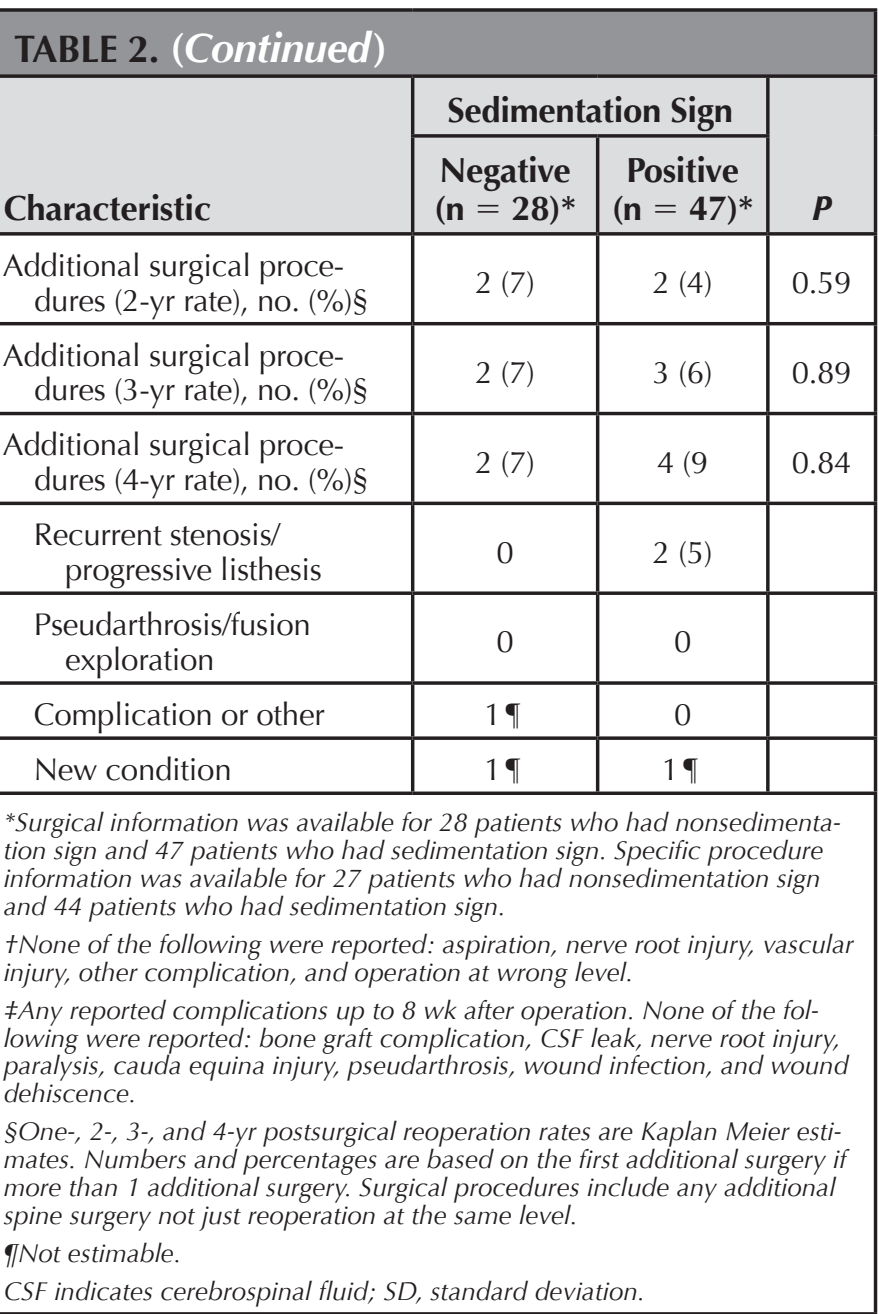

symptom severity or treatment outcome. This study evaluated the association between the recently described sedimentation sign and treatment outcomes in a cohort of patients enrolled in SPORT with LSS. We found that in these patients, a positive sedimentation sign was more often associated with central stenosis, multilevel stenosis, more proximal stenoses, and more severe degrees of canal narrowing; however, after adjusting for these traditional measures of stensosis severity along with demographic factors, we found that the group with a positive sedimentation sign displayed a somewhat greater surgical treatment effect for ODI. Thus, the sedimentation sign may be a useful adjunct for physicians and patients in making informed treatment choices for LSS. Although both subgroups showed a positive treatment effect from surgery, those with a positive sedimentation sign showed a marginally increased benefit from surgery that was significant for the ODI, although not significantly different for general pain or physical function; thus the presence or absence of the sedimentation sign could potentially help sway the balance of risks and benefits in patients who are uncertain about their preferred treatment choice. 


\begin{tabular}{|c|c|c|c|c|c|}
\hline Outcome & $\begin{array}{c}\text { Nerve Root } \\
\text { Sedimentation Sign }\end{array}$ & $\begin{array}{l}\text { Surgical, } \\
\text { Mean (SE) }\end{array}$ & $\begin{array}{c}\text { Nonoperative, } \\
\text { Mean (SE) }\end{array}$ & $\begin{array}{l}\text { Treatment Effectt } \\
\qquad(95 \% \mathrm{CI})\end{array}$ & $\boldsymbol{P}$ \\
\hline SF-36 bodily pain & Negative sedimentation sign & $26.3(3.9)$ & $14.8(4.6)$ & $11.5(3.2-19.9)$ & 0.007 \\
\hline \multirow{2}{*}{ (BP) $(0-100) \ddagger$} & Positive sedimentation sign & $26.4(3)$ & $10.9(3.1)$ & $15.5(9.1-21.8)$ & $<0.001$ \\
\hline & $P$ & 0.98 & 0.50 & 0.44 & \\
\hline SF-36 physical function & Negative sedimentation sign & $21.1(4.1)$ & $6(4.7)$ & $15.1(6.9-23.3)$ & $<0.001$ \\
\hline \multirow{2}{*}{ (PF) (0-100)‡ } & Positive sedimentation sign & $21(3.1)$ & $3.3(3.2)$ & $17.7(11.5-23.8)$ & $<0.001$ \\
\hline & $P$ & 0.99 & 0.65 & 0.61 & \\
\hline Oswestry Disability Index & Negative sedimentation sign & $-16.2(3)$ & $-9.1(3.5)$ & $-7(-13.3$ to -0.8$)$ & 0.028 \\
\hline \multirow{2}{*}{ ODI $(0-100) \S$} & Positive sedimentation sign & $-20.4(2.2)$ & $-4.4(2.4)$ & $-16(-20.8$ to -11.1$)$ & $<0.001$ \\
\hline & $P$ & 0.28 & 0.28 & 0.023 & \\
\hline $\begin{array}{l}\text { Stenosis bothersomeness } \\
\text { index }\end{array}$ & Negative sedimentation sign & $-6.1(1)$ & $-2.9(1.2)$ & $-3.2(-5.6$ to -0.8$)$ & 0.01 \\
\hline \multirow{2}{*}{$(0-24)$ ฯ } & Positive sedimentation sign & $-7.5(0.8)$ & $-1.9(0.8)$ & $-5.7(-7.6$ to -3.7$)$ & $<0.001$ \\
\hline & $P$ & 0.25 & 0.49 & 0.094 & \\
\hline \multicolumn{6}{|c|}{ 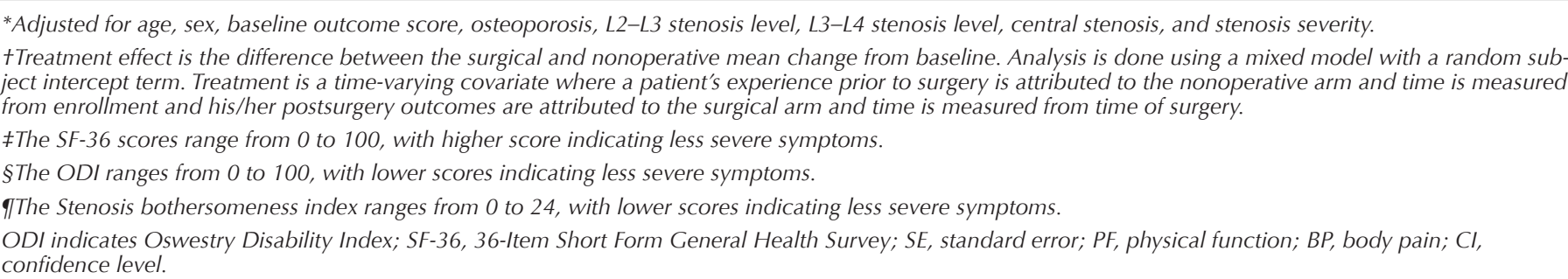 } \\
\hline
\end{tabular}

Several studies have evaluated MRI characteristics that may be associated with LSS severity and treatment effect, most notably, the dural sac cross-sectional area. However, these studies failed to find a significant relationship between anatomical narrowing, clinical severity, and treatment effect. $5,7,8,11,15,18,19,22$ Earlier studies revealed not only a wide range of results for both surgical and nonoperative interventions, but also failed to find a significant predictive characteristic. For example, Amundsen et al $l^{5,6}$ conducted a prospective study evaluating the imaging characteristics associated with LSS and found no correlation between the degree of dural sac narrowing and clinical disability or treatment effect.

Several other findings of our study are consistent with prior studies. A positive sedimentation sign was strongly associated with central stenosis, whereas the negative sedimentation sign group was more likely to have only lateral recess or neuroforaminal stenosis; similarly, a positive sedimentation sign was associated with multiple stenotic levels $(86 \%$ vs. $46 \%$ in the negative sedimentation sign group, $P=0.01) .{ }^{23,35}$ Because Barz et $a l^{23}$ only recently introduced the sedimentation sign, there are not many directly comparable studies that evaluated the association between the sedimentation sign and treatment outcomes. The observational study by Barz et $a^{25}$ showed that the sedimentation sign does not seem to predict surgical outcome, whereas in nonoperatively treated patients a positive sign was associated with a limited treatment outcome.

This study faced several limitations. This was a post hoc subgroup analysis from the SPORT study and used an observational, as-treated analysis due to high levels of treatment crossover in the randomized groups. However, we did use longitudinal mixed-models to attempt to control for confounding by any baseline differences between the subgroups. Furthermore, although excluded in the initial study by Barz et $a l,{ }^{23}$ patients who had LSS at L5-S1 were included in this study. However, there were only 4 patients who had only single-level L5-S1 stenosis, and on further analysis, only one of these had a positive sedimentation sign. Although the treatment effect results were consistent for the main outcome measures, they were statistically significant only for ODI and as such must be interpreted cautiously. It is worth noting, however, that the 2 measures (ODI and SBI) with the more suggestive findings were condition-specific outcome measure, which may be more responsive than the more general SF-36 measures. Also, because this was a subgroup analysis using previously collected and archived MRIs, our sample size and thus the power to detect a difference was somewhat limited. 
We also cannot be sure that the subgroup for whom complete imaging was available is representative of the overall stenosis cohort; prior studies with this data, however, suggest that measure demographic and imaging characteristics were similar between the groups with and without archived imaging data. ${ }^{27}$ Furthermore, we relied on clinically obtained images with varying image acquisition protocols, field strength, slice orientation, etc. This heterogeneity of imaging characteristics may have limited our ability to fully assess the association between sedimentation sign and treatment outcome.

This study employed an innovative approach by linking the assessment of a new diagnostic test with the evaluation of treatment effects. This type of test evaluation has been referred to as "clinical test validation" 24,36 providing direct evidence about the clinical meaning of test results, which is far more relevant information for clinicians than measures of test accuracy alone (e.g., test sensitivity and specificity). SPORT offers a unique opportunity to evaluate emerging radiological tests, because the archived MR images can be revisited when new radiological criteria like the sedimentation sign are discovered. It would be desirable if establishing trial-based digital archives of imaging data became standard practice in the conduct of treatment trials to enable test evaluations such as the one presented here.

\section{CONCLUSION}

In this preliminary study, we demonstrated that controlling for other demographic and imaging features, a positive sedimentation sign was associated with a somewhat greater surgical treatment effect in patients with symptomatic LSS for ODI, though not for other measured outcomes. These findings suggest that a positive sedimentation sign may be useful as an adjunct to help guide an informed treatment choice regarding surgery for patients with symptomatic LSS. The presence or absence of the sedimentation sign, which seems to help predict the size of the surgical treatment after adjusting for other traditional measures of stenosis severity, may help sway the balance of risks and benefits in patients who are uncertain about their preferred treatment choice.

\section{Key Points}

LSS is one of the most common reasons for surgery in the US elderly, but there is a dearth of reliable diagnostic tools that give a clear indication for surgery.

T2-weighted images of patients with LSS enrolled in SPORT were analyzed by an independent orthopedic surgeon for sedimentation sign.

- Also, $66 \%$ of the 115 patients whose images were analyzed were found to have a positive sedimentation sign.

Surgical treatment effects for those patients with positive sedimentation sign were somewhat larger in all outcomes, and significant for ODI $(-16$ vs. $-7 ; P=0.02)$, after adjusting for other demographic and imaging features.

\section{References}

1. Verbiest H. A radicular syndrome from developmental narrowing of the lumbar vertebral canal. 1954. Clin Orthop Relat Res 2001;384:3-9.

2. Ciol MA, Deyo RA, Howell E, et al. An assessment of surgery for spinal stenosis: time trends, geographic variations, complications, and reoperations. J Am Geriatr Soc 1996;44:285-90.

3. Katz JN, Harris MB. Clinical practice. Lumbar spinal stenosis. $N$ Engl J Med 2008;358:818-25.

4. Aalto TJ, Malmivaara A, Kovacs F, et al. Preoperative predictors for postoperative clinical outcome in lumbar spinal stenosis: systematic review. Spine (Phila Pa 1976) 2006;31:E648-63.

5. Amundsen T, Weber H, Lilleas F, et al. Lumbar spinal stenosis. Clinical and radiologic features. Spine (Phila Pa 1976) 1995;20: 1178-86.

6. Amundsen T, Weber H, Nordal HJ, et al. Lumbar spinal stenosis: conservative or surgical management?: A prospective 10-year study. Spine (Phila Pa 1976) 2000;25:1424-35; discussion 35-6.

7. Fritz JM, Delitto A, Welch WC, et al. Lumbar spinal stenosis: a review of current concepts in evaluation, management, and outcome measurements. Arch Phys Med Rehabil 1998;79:700-8.

8. Herno A, Airaksinen O, Saari T, et al. Lumbar spinal stenosis: a matched-pair study of operated and nonoperated patients. $\mathrm{Br} J$ Neurosurg 1996;10:461-5.

9. Herno A, Airaksinen O, Saari T, et al. The effect of prior back surgery on surgical outcome in patients operated on for lumbar spinal stenosis. A matched-pair study. Acta Neurochir (Wien) 1996;138:357-63.

10. Sirvanci M, Bhatia M, Ganiyusufoglu KA, et al. Degenerative lumbar spinal stenosis: correlation with Oswestry Disability Index and MR imaging. Eur Spine J 2008;17:679-85.

11. Barz T, Melloh M, Staub L, et al. The diagnostic value of a treadmill test in predicting lumbar spinal stenosis. Eur Spine $J$ 2008;17:686-90.

12. Deen HG, Jr, Zimmerman RS, Lyons MK, et al. Measurement of exercise tolerance on the treadmill in patients with symptomatic lumbar spinal stenosis: a useful indicator of functional status and surgical outcome. J Neurosurg 1995;83:27-30.

13. Deen HG, Zimmerman RS, Lyons MK, et al. Use of the exercise treadmill to measure baseline functional status and surgical outcome in patients with severe lumbar spinal stenosis. Spine (Phila Pa 1976) $1998 ; 23: 244-8$.

14. Jane JA, Sr, Jane JA, Jr, Helm GA, et al. Acquired lumbar spinal stenosis. Clin Neurosurg 1996;43:275-99.

15. Katz JN, Dalgas M, Stucki G, et al. Degenerative lumbar spinal stenosis. Diagnostic value of the history and physical examination. Arthritis Rheum 1995;38:1236-41.

16. Hamanishi C, Matukura N, Fujita M, et al. Cross-sectional area of the stenotic lumbar dural tube measured from the transverse views of magnetic resonance imaging. J Spinal Disord 1994;7:388-93.

17. Ogikubo O, Forsberg L, Hansson T. The relationship between the cross-sectional area of the cauda equina and the preoperative symptoms in central lumbar spinal stenosis. Spine (Phila Pa 1976) 2007;32:1423-8; discussion 9.

18. Boswell MV, Trescot AM, Datta S, et al. Interventional techniques: evidence-based practice guidelines in the management of chronic spinal pain. Pain Physician 2007;10:7-111.

19. Gibson JN, Waddell G. Surgery for degenerative lumbar spondylosis: updated Cochrane Review. Spine (Phila Pa 1976) 2005;30:2312-20.

20. Gibson JN, Waddell G. Surgery for degenerative lumbar spondylosis. Cochrane Database Syst Rev 2005;30:CD001352.

21. Konno S, Kikuchi S, Tanaka Y, et al. A diagnostic support tool for lumbar spinal stenosis: a self-administered, self-reported history questionnaire. BMC Musculoskelet Disord 2007;8:102.

22. Konno S, Hayashino Y, Fukuhara S, et al. Development of a clinical diagnosis support tool to identify patients with lumbar spinal stenosis. Eur Spine J 2007;16:1951-7.

23. Barz T, Melloh M, Staub LP, et al. Nerve root sedimentation sign: evaluation of a new radiological sign in lumbar spinal stenosis. Spine (Phila Pa 1976) 2010;35:892-7.

www.spinejournal.com

135 
24. Staub LP, Barz T, Melloh M, et al. Clinical validation study to measure the performance of the nerve root sedimentation sign for the diagnosis of lumbar spinal stenosis. Contemp Clin Trials 2009;32:470-4.

25. Barz T, Staub LP, Melloh M, et al. Clinical validity of the nerve root sedimentation sign in patients with suspected lumbar spinal stenosis. Spine J 2013;14:667-74.

26. Weinstein JN, Tosteson TD, Lurie JD, et al. Surgical versus nonsurgical therapy for lumbar spinal stenosis. N Engl J Med 2008;358:794-810.

27. Lurie JD, Tosteson AN, Tosteson TD, et al. Reliability of readings of magnetic resonance imaging features of lumbar spinal stenosis. Spine (Phila Pa 1976) 2008;33:1605-10.

28. Birkmeyer NJ, Weinstein JN, Tosteson AN, et al. Design of the Spine Patient outcomes Research Trial (SPORT). Spine (Phila Pa 1976) 2002;27:1361-72.

29. Cummins J, Lurie JD, Tosteson TD, et al. Descriptive epidemiology and prior healthcare utilization of patients in the Spine Patient Outcomes Research Trial's (SPORT) three observational cohorts: disc herniation, spinal stenosis, and degenerative spondylolisthesis. Spine (Phila Pa 1976) 2006;31:806-14.
30. Ware JE, Jr, Sherbourne CD. The MOS 36-Item Short-Form Health Survey (SF-36). I. Conceptual framework and item selection. Med Care 1992;30:473-83.

31. McHorney CA, Ware JE, Jr, Lu JF, et al. The MOS 36-Item ShortForm Health Survey (SF-36): III. Tests of data quality, scaling assumptions, and reliability across diverse patient groups. Med Care 1994;32:40-66.

32. Patrick DL, Deyo RA, Atlas SJ, et al. Assessing health-related quality of life in patients with sciatica. Spine (Phila Pa 1976) 1995;20:1899-908; discussion 909.

33. Atlas SJ, Deyo RA, Keller RB, et al. The Maine Lumbar Spine Study, Part III. 1-year outcomes of surgical and nonsurgical management of lumbar spinal stenosis. Spine (Phila Pa 1976) 1996;21:1787-94; discussion 94-5.

34. Fairbank JC, Couper J, Davies JB, et al. The Oswestry Low Back Pain Disability Questionnaire. Physiotherapy 1980;66:271-3.

35. Macedo LG, Wang Y, Battie MC. The sedimentation sign for differential diagnosis of lumbar spinal stenosis. Spine (Phila Pa 1976) 2012;38:827-31.

36. Reitsma JB, Rutjes AW, Khan KS, et al. A review of solutions for diagnostic accuracy studies with an imperfect or missing reference standard. J Clin Epidemiol 2009;62:797-806. 